\title{
Genotyping for disease associated HLA DR $\beta 1$ alleles and the need for early joint surgery in rheumatoid arthritis: a quantitative evaluation
}

\author{
Anne Crilly, Nicola Maiden, Hilary A Capell, Rajan Madhok
}

\begin{abstract}
Objective-To determine the value of HLA DR $\beta 1$ disease associated epitope (DAE) and erythrocyte sedimentation (ESR) in predicting the need for major joint replacement in rheumatoid arthritis (RA).

Methods-Sixty five RA patients who had undergone hip, knee or shoulder arthroplasty within 15 years of disease onset and 65 who had not. HLA DR $\beta 1$ genotype was determined by polymerase chain reaction. ESR at first hospital visit was noted.

Results-Significantly more patients with two DAE required surgery, (32\% $v 9 \%), \chi^{2}$ $=13.9, p=0.001$, odds ratio $=5.4(95 \% \mathrm{CI}$ : $1.8,16)$. Sensitivity was poor, $32 \%$, specificity high, 91\%. Presentation ESR was higher in surgery patients compared with non-surgery patients, $52 \mathrm{~mm} 1$ st h $v 25$ $\mathrm{mm} 1$ st $h, p<0.001$, but was independent of DAE status. Sensitivity of an ESR of 30 mm 1st h was $75 \%$, specificity $53 \%$.

Conclusion-The presence of two DAE is a risk factor for major joint surgery in RA and is independent of ESR, whereas in those with one or no DAE, a high ESR is an important predictor.

(Ann Rheum Dis 1999;58:114-117)
\end{abstract}

Joint destruction because of rheumatoid arthritis (RA) means pain, disability, and socioeconomic cost. The ability to predict future destructive disease at onset is an important but yet unrealised goal. In these patients there is a narrow therapeutic window between the stage of persistent synovitis and irreversible cartilage loss. ${ }^{1}$ It is during this interval that disease modifying drugs may confer additional advantage. These treatments however are potentially toxic and require close monitoring.

Several predictors of outcome are recognised, which although useful in epidemiological studies are of limited value in routine clinical practice. Serum rheumatoid factor is useful in diagnosis but is not discriminatory enough to accurately identify individual patients with a poor prognosis. ${ }^{2}$ Clinical and laboratory indices of inflammation such as erythrocyte sedimentation rate (ESR) and $\mathrm{C}$ reactive protein $(\mathrm{CRP})$ improve with disease modifying treatment but are also influenced by age, coexisting disease and occasionally may be normal in the presence of active disease. Baseline ESR is also a useful indicator of radiological progression over a 5 and 10 year follow up, ${ }^{3}$ although the precise value of ESR at presentation as a long term prognostic marker is not accurately quantified. The number of involved joints at presentation is a predictor of mortality. In those with 30 or more joints involved at presentation, five year survival was less than $50 \%{ }^{4}$

HLA DR $\beta 1$ alleles associated with RA share sequence homology within the third hypervariable region. ${ }^{5}$ Phenotypic studies have provided suggestive but not conclusive evidence for more severe disease in HLA DR4 positive patients. ${ }^{6}$ Genotypic studies for HLA DR $\beta 1$ alleles are cheaper, more accurate and informative. In RA it has been suggested that the gene dose results in a hierarchical outcome, one disease epitope resulting in erosive disease and two predisposes to more severe articular as well as extra-articular disease. ${ }^{7}$ Theses alleles include HLA DR $\beta 1{ }^{\star} 0401,0404,0405,0408$, $1402,1001,0101$, and $0102 .^{8}$

The aim of this study was to accurately quantify the value of genotyping for the disease epitope in identifying those with more severe disease as defined by the need for either shoulder, hip or knee arthroplasty within 15 years of disease onset.

\section{Methods}

SELECTION OF CASE PATIENTS AND CONTROL SUBJECTS

A retrospective case-series study was performed to determine the relation between HLA genotype and need for joint surgery before 15 years of disease duration. In a previous survey of 100 consecutive RA patients who had undergone joint replacement surgery attending the same clinic, the median disease duration before requiring surgery was 14.6 years. All study patients were white. One hundred and thirty RA patients consecutively seen by one doctor who satisfied the study entry criteria between March 1995 and April 1996 were included. They were selected from a cohort of 1512 patients attending a hospital based clinic for RA patients. All study patients fulfilled the 
Table 1 Demographic and clinical characteristics of patients who did and did not require joint surgery before 15 years

\begin{tabular}{llllllll}
\hline Surgery & Age $(y)$ & Sex M:F & $\begin{array}{l}\text { Disease duration } \\
(y)\end{array}$ & $\begin{array}{l}\text { Age at disease } \\
\text { onset }(y)\end{array}$ & HAQ & DMARD & ESR (mm 1st hr) \\
\hline Yes $(\mathrm{n}=65)$ & $59(51-68)$ & $15: 50$ & $\star \star 15(10-17)$ & $43(37-54)$ & $\star 2.4(2-2.6)$ & $3(2-5)$ & $\star \star 52.5(31-76)$ \\
No $(\mathrm{n}=65)$ & $63(58-69)$ & $11: 54$ & $20(18-24)$ & $41(34-48)$ & $2(1.5-2.5)$ & $2(1-4)$ & $25.5(15-43)$ \\
\hline
\end{tabular}

Median values are shown with interquartile ranges given in parentheses. $M=$ male; $F=$ female; HAQ $=$ Health Assessment Questionnaire; DMARD = disease modifying anti-rheumatic drugs; ESR = erythrocyte sedimentation rate. ${ }^{\star \star} \mathrm{p}<0.001,{ }^{\star} \mathrm{p}<0.01$.

1987 American College of Rheumatology criteria for RA, ${ }^{9}$ were seropositive for rheumatoid factor and had erosive disease. Patients were selected if they had required either shoulder, hip or knee arthroplasty within 15 years of disease $(n=65)$. Patients with a disease duration greater than 15 years who had not required surgery served as controls $(n=65)$. The extent of disability at the time of study was determined using the Health Assessment Questionnaire (HAQ). ${ }^{10}$

DETERMINATION OF HLA GENOTYPES

Genomic DNA was extracted from venous blood collected in EDTA (Genomix Kit, VH Bio, Newcastle Upon Tyne, UK). Sequence specific primers (SSP) to the HLA DR $\beta 1$ genes that amplified the alleles 1-16 and 51-53 were used (BSHI, University of Bristol, Bristol UK). Amplification was performed in a final volume of $10 \mu \mathrm{l}(1 \times$ polymerase chain reaction (PCR) buffer, $1.5 \mathrm{mM} \mathrm{MgCl}_{2}, 200 \mu \mathrm{M}$ dNTPs, primers, 0.125 units of Taq polymerase, $80 \mathrm{ng}$ of genomic DNA). The cycling parameters used for the reaction were those advised by the manufacturer. Patients who were DR4 and DR1 positive were subtyped using SSP primers (Dynal, UK). Genomic DNA (50 ng/ $\mu \mathrm{l})$ was used as a template for amplification with 12 sets of primers to amplify DR $\beta 1^{\star} 0401$ to ${ }^{\star} 04019$ and five sets of primers to amplify DR $\beta 1^{\star} 0101$ to ${ }^{\star} 0104$ as advised by the manufacturer. The PCR products were run on a $2 \%$ agarose gel and visualised by ethidium bromide staining. A kit containing DR $\beta 1$ control DNA ((type 2/96), UKTSSA, Bristol) was used to check specificity. Disease associated alleles that give rise to the disease associated epitope (DAE) included HLA DR $\beta 1{ }^{\star} 0401$, * $0404,{ }^{\star} 0405,{ }^{\star} 0408,{ }^{\star} 0101,{ }^{\star} 0102,{ }^{\star} 1001$.

Table 2

A

\begin{tabular}{lllll}
\hline Allele observed & Surgery & No surgery & Total & $\%$ \\
\hline Numbers of people carrying alleles that give rise to disease & associated epitopes & \\
DR $\beta 1^{\star} 0101$ & 14 & 9 & 23 & 18 \\
DR $\beta 1^{\star} 0401$ & 24 & 17 & 41 & 32 \\
DR $\beta 1^{\star} 0404$ & 17 & 5 & 22 & 17 \\
DR $\beta 1^{\star} 0405$ & 0 & 2 & 2 & 1.5 \\
DR $\beta 1^{\star} 0408$ & 6 & 3 & 9 & 7 \\
DR $\beta 1^{\star} 10$ & 2 & 0 & 2 & 1.5 \\
\hline
\end{tabular}

B

\begin{tabular}{lcccc}
\hline Allele combination & Surgery $(n=65)$ & No surgery $(n=65)$ & Total $(n=130)$ & $\%$ \\
\hline \multicolumn{2}{l}{ Summary of people carrying two alleles that give rise to the disease asssociated epitope } & \\
${ }^{\star} 0101 / 0101$ & 2 & 1 & 3 & 2 \\
${ }^{\star} 0101 / 0401$ & 5 & 1 & 6 & 5 \\
${ }^{\star} 0401 / 0401$ & 3 & 2 & 5 & 4 \\
${ }^{\star} 0401 / 0404$ & 4 & 0 & 4 & 3 \\
${ }^{\star} 0401 / 0408$ & 2 & 0 & 2 & 1.5 \\
${ }^{\star} 0404 / 0408$ & 3 & 1 & 4 & 3 \\
${ }^{\star} 0404 / 0405$ & 0 & 1 & 1 & 0.8 \\
$10 / 10$ & 1 & 0 & 1 & 0.8 \\
\hline
\end{tabular}

ERYTHROCYTE SEDIMENTATION RATE

When available the initial ESR at first ever visit to the outpatient department at Glasgow Royal Infirmary with presentation of disease was recorded. The ESR had been measured by the Westergren method.

STATISTICAL ANALYSIS

To show a $40 \%$ difference $(\alpha=0.02, \beta=0.2)$ 30 patients were required in each group. To correct for potential confounding variables 65 patients per group were recruited. Medians and interquartile ranges are reported. Continuous data were analysed by the Mann-Whitney test, discrete data were analysed by $\chi^{2}$ using $2 \times$ 2 contingency tables. $\mathrm{p}$ Values $<0.05$ were taken as being significant. To determine the combined influence of HLA genotype and ESR on outcome, logistic regression analysis was performed. In the regression model ESR was a continuous variable.

\section{Results}

DEMOGRAPHIC AND CLINICAL CHARACTERISTICS OF THE RA PATIENTS

Table 1 shows the demographic and disease characteristics of the surgery $(n=65)$ and nonsurgery $(n=65)$ patients. In the non-surgery group, disease duration was longer than in the surgery group $(\mathrm{p}<0.0001)$. The median disease duration at time of surgery was 8.5 years (IQR: 6-11 years). HAQ score was significantly worse in the surgery group $(\mathrm{p}=$ 0.001 ), but its clinical significance is unclear. In no patient was there evidence of osteoarthritis at disease onset.

DISTRIBUTION OF HLA DR $\beta 1$ ALLELES

The alleles known to code for DAE were observed in $84(65 \%)$ of the $130 \mathrm{RA}$ patients recruited. The alleles that give rise to DAE that were observed in our study population (both cases and controls) included DR $\beta 1^{\star}$ 0401, ${ }^{\star} 0404,{ }^{\star} 0405,{ }^{\star} 0408,{ }^{\star} 0101$, and DR $\beta 1{ }^{\star} 10$, as shown in table $2 \mathrm{~A}$.

Twenty seven patients had two DAE, the alleles found included: DR $\beta 1^{\star} 0401 / 0101$, 0401/0401, 0401/0404, 0401/0408, 0404/ 0408, 0101/0101, see table 2B. Three patients carried either $0408 / 0408 ; 1001 / 1001$ or $0404 /$ 0405.

Significantly more patients in the surgery group carried 2 DAE compared with the nonsurgery group, $32 \% v 9 \% \chi^{2}=13.9,2 \mathrm{df}$, $\mathrm{p}=0.001$. The sensitivity and specificity of having two DAE present for predicting requirement for surgery was $32 \%$ and $91 \%$ respectively, odds ratio (OR) was 5. Comparing those with two DAE with those who had no DAE, OR was 7 and the test sensitivity $58 \%$ while specificity was $84 \%$. There was no statistical 
Table 3 Logistic regression analysis relating HLA status (2DAE) and baseline ESR on requirement for surgery

\begin{tabular}{llll}
\hline Variable & Regression coefficient & Standard error & Significance \\
\hline Two DAE & 1.6877 & 0.5569 & 0.0024 \\
ESR & 0.0343 & 0.0085 & 0.001 \\
Constant & -1.776 & 0.4226 & $<0.0001$ \\
\hline
\end{tabular}

Logit $(\mathrm{P})$ or $\mathrm{l}=-1.776+1.6877 \mathrm{x}_{1}+0.0343 \mathrm{x}_{2}$, were $\mathrm{x}_{1}$ is the presence $(\mathrm{x}=1)$ or absence $(\mathrm{x}=2)$ of 2 DAE; $x_{2}$ is the ESR at first clinic visit; $P=$ probability of surgery.

difference in need for surgery between those with one DAE and no DAE ( $66 \%$ v $47 \%, \chi^{2}=$ $3.5,1 \mathrm{df} p=0.06)$.

ERYTHROCYTE SEDIMENTATION RATE

One hundred and twenty four of the 130 patients had an ESR recorded at the first clinic visit. The ESR was significantly higher in the surgery ( $\mathrm{n}=62$, median: $52.5 \mathrm{~mm} \mathrm{1st} \mathrm{h,} \mathrm{IQR:}$ 31-76) compared with the non-surgery group ( $\mathrm{n}=62$, median: $25.5 \mathrm{~mm}$ 1st h, IQR: $15-43$ ), p $<0.001$. A ROC curve analysis (data not shown) showed that an ESR of $30 \mathrm{~mm} 1 \mathrm{st} \mathrm{h}$ was the most discriminatory cut off value. An ESR of $30 \mathrm{~mm} 1 \mathrm{st} \mathrm{h}$ had a high sensitivity of $74 \%$ but a low specificity, $53 \%$ in identifying those requiring early surgery.

When all patients were considered, those with two DAE ( $\mathrm{n}=27)$ had a median ESR of 43 mm 1st h (IQR: 15-86) and those with either one DAE or none ( $\mathrm{n}=97)$, had a median ESR of $37 \mathrm{~mm} \mathrm{1st} \mathrm{h} \mathrm{(IQR:} \mathrm{21-58),} \mathrm{p} \mathrm{>} \mathrm{0.05,}$ indicating that ESR was independent of HLA status.

PREDICTING SURGERY USING HLA STATUS AND

ESR

To predict the need for surgery within 15 years of disease onset using HLA and baseline ESR, a model using logistic regression analysis was derived. The full model is shown in table 3 and confirms that ESR and HLA predict need for surgery independently of each other. Considering HLA and ESR independently the need for early surgery was predicted in $62 \%$ and $68 \%$ of patients respectively, however when combined the need for surgery was predicted in $73 \%$.

The log probability of surgery, logit $(\mathrm{P})$ or 1 was calculated using the equation:

$1=-1.776+1.6877 \mathrm{x}_{1}+0.0343 \mathrm{x}_{2}\left[\mathrm{x}_{1}\right.$ is HLA status $\left(x_{1}=1\right.$ if 2 DAE present, $x_{1}=0$ if 1 or no DAE present) and $x_{2}$ is ESR at first clinic visit]. The probability of surgery can be calculated using the equation: $\mathrm{P}=\mathrm{e}^{1} / 1+\mathrm{e}^{1}$

By setting $\mathrm{x}_{1}=1$ or $\mathrm{x}_{2}=0$, we generated two equations, 1 (1) and 1 (0) from which the log odds was calculated: $1(1)-1(0)=1.6877$, giving an odds ratio of 5.4 (95\% CI: 1.8, 16). Thus those with $2 \mathrm{DAE}$ were 5.4 times more likely to require early surgery compared with those with 1 or no DAE, when ESR is not taken into account.

The odds ratio for surgery in those with two DAE did not change with increasing ESR, whereas the odds ratio increased from 1 (95\% CI: $0.6,3.1)$ at an ESR of $30 \mathrm{~mm} 1 \mathrm{st}$ h to 2 (95\% CI: $0.8,4)$ at an ESR of $60 \mathrm{~mm} 1 \mathrm{st} \mathrm{h}$ in those with one or no DAE. Thus in patients with one or no DAE the risk for surgery increases with increasing ESR. The influence of one DAE was not examined.

\section{Discussion}

We show that RA patients homozygous for the DAE are five times more likely to require major joint surgery compared with those with none. As a predictive test we calculated that in those homozygous for the disease epitope the false positive rate was $9 \%$ and false negative rate $68 \%$. ESR at presentation gave a false positive rate of $47 \%$ and a false negative rate of $24 \%$. We also found that the need for surgery seemed to be directly proportional to the ESR in those with one or no DAE but not in those with two DAE.

Weyand et al found that $60 \%$ of RA patients homozygous for the disease epitope required a joint replacement, compared with $25 \%$ of heterozygotes. $^{8}$ This study was in a cohort attending a tertiary referral centre of which only four patients lacked a disease associated allele. Eberhardt et al also found that the need for surgery was three times greater in their homozygous group. ${ }^{11}$ The numbers were small and in the latter study duration follow up short. The findings in both may have arisen because of bias or statistical error. In neither could the value of the test in clinical practice be accurately quantified. The importance of ESR was not considered in either study.

These important preliminary results lead us to undertake this more specific study in which we made three assumptions. Firstly, at least a $40 \%$ difference between the two groups was considered necessary in calculating study size. This difference was deemed appropriate for any future prospective therapeutic study if more aggressive treatments were to be based on this test. It also gave a feasible study size. To further increase the power of the study only patients under the care of one consultant were included. Secondly, although we tested for a large number of alleles we made no correction for the number of alleles as the original hypothesis was based on either the presence or absence of the disease epitope. Finally, although other studies observed differences in the relative effects of the epitopes we assumed a similar influence of each.

Previous studies have demonstrated a direct association between ESR and radiological progression of disease. ${ }^{4}{ }^{12} \mathrm{Fex}$ et al $\mathrm{l}^{12}$ reported that a high baseline ESR was one of several factors that predicted radiological progression in $57 \%$ of patients. In our study the RA patients who required surgery had a median ESR twice that of RA controls, although there was considerable overlap. Using initial ESR and DAE status allows surgery before 15 years duration to be correctly predicted in $73 \%$ of patients. This was compared with $62 \%$ and $68 \%$ correctly predicted when using HLA status or ESR respectively on their own. Thus prediction for surgery is improved by considering both HLA status and baseline ESR. In those with no or one DAE the need for surgery increases in direct proportion to ESR, while in those with two DAE the need is independent of the acute phase response.

Joint surgery was chosen as an end point because it was a well defined and easily quantified outcome and previous studies evaluating 
DAE as a predictor had used this parameter. An alternative would have been disability. Serological studies of HLA show DR4 to be associated with a higher radiographic score and rheumatoid factor positivity but not with a worse functional status. ${ }^{13}$ Studies examining the influence of the genotype in predicting disability as assessed by disability have been conflicting. In two, inflammatory polyarthritis rather than RA was studied and divergent results were obtained. In both the primary aim was to establish the value of the test in predicting $\mathrm{RA}$ rather than HAQ score. ${ }^{14}{ }^{15}$ In the two RA studies, one reported an association and in the other the numbers were too small for confident statistical analysis and period of observation not long enough. ${ }^{16}{ }^{17}$ Disability therefore appears to measure an aspect of the pathogenetic mechanism that is independent of the HLA status. We found that patients who required surgery were significantly more disabled, however no firm conclusion can be drawn and further studies specifically on this issue are necessary.

Few predictive tests of long term outcome in $\mathrm{RA}$ are available. The presence of two DAE is a predictor for major joint surgery in RA and is independent of ESR, whereas in patients with one or no DAE, a high ESR is a better indicator in our study. As treatments for RA continue to improve stratifying patients before joint damage being manifest will become increasingly important. We suggest that genotyping for the disease epitope is a useful test for selecting those likely to require early major joint surgery.

We are grateful to The Robertson Trust (Glw) for financial support. Dr Maiden is a BUPA/ Glasgow Royal College of port. Dr Maiden is a BUPA/ Glasgow
Physicians and Surgeons Research Fellow. 1 Fuchs, HA, Kay JJ, Callahan LF, Nance EP, Pincus J. arthritis within first 2 years of disease. J Rheumatol 1989;16:585-91.
2 Eberhardt KB, Svenson B, Truedsson L, Wolheim FA. The occurrence of rheumatoid factor isotypes in early definite heumatoid arthritis-no relationship with erosions or disease activity. J Rheumatol 1988;15:1070-4.

3 Capell HA, Murphy EA, Hunter JA. Rheumatoid arthritis: workload and outcome over 10 years. Q J Med 1991;79: 461-76.

4 Pincus T, Brooks RN, Callahan LF. Prediction of long term mortality in patients with rheumatoid arthritis according to a simple questionnaire and joint count measures. Ann Intern Med 1984;120:26-34.

5 Gregersen PK, Silver J, Winchester RJ. The shared epitope hypothesis. An approach to understanding the molecular genetics of susceptibility to rheumatoid arthritis. Arthritis Rheum 1987;30:1205-13.

6 Calin A, Elswood J, Klouda PT. Destructive arthritis, rheumatoid factor and HLA DR4. Arthritis Rheum 1989;3: 1221-5.

7 Weyand CM, McCarthy TG, Goronzy JJ. Correlation between disease phenotype and genetic heterogeneity in rheumatoid arthritis. J Clin Invest 1995;95:2120-6.

8 Weyand CM, Hicok KC, Conn D, Goronzy JJ. The influence of HLA DR $\beta 1$ genes on disease severity in rheumatoid arthritis. Ann Intern Med 1992;1 17:801-6.

9 Arnett FC, Edworthy SM, Bloch DA, McShane DJ, Fries JF, Cooper NS, et al. The American Rheumatism Association 1987 revised criteria for the classification of rheumatoid arthritis. Arthritis Rheum 1988;31:315-24.

10 Fries, JF, Spitz PW, Kraines RG. Measurement of patient outcome in arthritis. Arthritis Rheum 1980;23:146-52.

11 Eberhardt K, Fex E, Johnson U, Wollheim FA. Association of HLA DR $\beta$ and DQ $\beta$ genes with two and five year outcome in rheumatoid arthritis. Ann Rheum Dis 1996;55: outco 34 .

12 Fex E, Jonsson K, Johnson U, Eberhardt K. Development of radiographic damage during the first 5-6 years of rheumatoid arthritis. A prospective follow-up study of a Swedish cohort. Br J Rheumatol 1996;35:1106-15.

13 Olsen NJ, Callahan LF, Brooks RH, Nance EP, Kaye JJ, Stastny P, et al. Association of HLA DR4 with rheumatoid factor and radiographic severity in rheumatoid arthritis. Am J Med 1988;84:257-64.

14 Brennan P, Silman A, Harrison B, Ollier B, Jawaheer D, Symmons D. The role of HLA DR $\beta 1$ genotype in predicting short term outcome of inflammatory polyarthritis. Arthritis Rheum 1995;38:S181.

15 Gough A, Faint J, Salmon M, Hassell A, Wordsworth P, Pilling D, et al. Genetic typing of patients with inflammatory arthritis at presentation can be used to predict tory arthritis at presentation can be used

16 Suarez-Almazor ME, Tao S, Moustarah F, Russell AS, Maksymowych W. HLA DR1, DR4 and DR $\beta 1$ related subtypes in rheumatoid arthritis. Association with susceptibility but not severity in a city wide community based study. J Rheumatol 1995;22:2027-33.

17 van Zeben D, Hazes JMW, Zwinderman AH, Cats A, Schreuder GM, D'Amaro J, et al. Association of HLA DR 4 with a more progressive disease course in patients with rheumatoid arthritis. Arthritis Rheum 1991;34:822-30. 